\title{
Biology and Treatment of Primary Central Nervous System Lymphoma
}

\author{
Alain P. Algazi, Cigall Kadoch, and James L. Rubenstein \\ Division of Hematology and Oncology, University of California, San Francisco, San Francisco, California 94143
}

\begin{abstract}
Summary: Primary central nervous system lymphoma (PCNSL) is a rare variant of extranodal non-Hodgkin lymphoma that is restricted in distribution to the brain, leptomeninges, spinal cord, and intraocular compartments. Although PCNSL shares overlapping features with systemic lymphoma, recent studies also reveal a unique pattern of gene and protein expression in PCNSL. These findings have yielded new insights into the pathophysiology of the disease, as well as the identification of novel prognostic biomarkers. Immune system compromise, such as is seen in acquired immune deficiency syndrome (AIDS), is the best established known risk factor for PCNSL. Like other lesions of the brain, meninges, and eye, the presenting symptoms associated with PCNSL typically include focal neurological deficits related to the site of disease or more global consequences of increased intracranial pressure. Diagnosis of PCNSL typically includes gadolinium-enhanced MRI and pathologic tissue analysis, as well as additional studies
\end{abstract}

aimed at excluding concurrent systemic disease. PCNSL typically has a worse overall prognosis than systemic lymphoma. High-dose chemotherapy, particularly with methotrexate-based regimens, is the backbone of therapy for most patients, and chemotherapy is associated with much lower rates of treatmentrelated morbidity and mortality than whole-brain irradiation. Autologous stem cell transplantation is an emerging treatment modality, particularly in younger patients with relapsed disease, but high rates of treatment-related mortality are observed in older patients. Immunotherapy, including treatment with intrathecal rituximab, is another area of active research that may have promise in refractory or relapsed disease. Treatment options for intraocular lymphoma parallel those for PCNSL elsewhere in the brain: systemic chemotherapy, radiation, and local delivery of cytotoxic and immunologically active agents such as anti-CD20 antibody. Key Words: Brain tumor treatment, gene expression, pathobiology, immunotherapy.

\section{INTRODUCTION}

Primary central nervous system lymphoma (PCNSL) is a rare variant of extranodal non-Hodgkin lymphoma (NHL) that is restricted in distribution to the brain, meninges, spinal cord, and eye. It represents $1 \%$ to $2 \%$ of all cases of $\mathrm{NHL}^{1}$ and $3.1 \%$ of primary brain tumors. ${ }^{2} \mathrm{Im}$ mune system compromise is the only known risk factor for the disease, and the incidence of PCNSL rose dramatically during the peak of the acquired immune deficiency syndrome (AIDS) epidemic in the late 1980s., Ninety percent of PCNSL lesions in patients with normal immune function exhibit diffuse large B-cell histologies, with the remainder exhibiting other B-cell or T-cell phenotypes; however, given the same histology, outcomes are generally worse in PCNSL than in systemic lym-

\footnotetext{
Address correspondence and reprint requests to: James L. Rubenstein, M.D., Ph.D., Division of Hematology and Oncology, University of California, San Francisco, 505 Parnassus Avenue, Suite M1286, Box 1270, San Francisco, CA 94143. E-mail: jamesr@medicine.ucsf.edu.
}

phoma. Furthermore, the major chemotherapeutic agents for systemic NHL, such as doxorubicin and cyclophosphamide, are ineffective in PCNSL. While whole-brain radiotherapy (WBRT) has been the historical cornerstone of treatment for this disease, new treatment approaches are emerging that are more effective and less toxic. A substantial proportion of PCNSL patients ultimately develop refractory, or recurrent disease. This review addresses progress to date in the diagnosis and treatment of PCNSL. A focus is on the identification of novel prognostic biomarkers, as well as biological clues that illuminate the pathophysiology of PCNSL and that may point to potentially significant therapeutic leads.

\section{PATHOLOGICAL FEATURES AND PROGNOSTIC BIOMARKERS}

PCNSL differs from systemic lymphoma at the cellular and molecular level. Immunophenotypic findings in B-cell lymphoma in the CNS show that these tumors share molecular features with multiple systemic B-cell 
lymphoma subtypes, but such shared molecular features do not necessarily mean that the tumors will exhibit similar clinical behavior.

It has been proposed that systemic large cell lymphomas can be classified into two major distinct subtypes: germinal center and activated B-cell. ${ }^{5}$ In the systemic setting, germinal B-cell lymphomas express BCL-6, and they tend to confer a more favorable prognosis than the other subtypes. In the CNS, large B-cell lymphoma also tends to express BCL-6. Furthermore sequencing of the immunoglobulin variable regions in these tumors reveals a pattern of frequent somatic mutation that suggests that they are derived from mature B-cells that have undergone T-cell-dependent affinity maturation in a germinal center microenvironment. ${ }^{6}$ However, PCNSL is associated with a worse prognosis than systemic large cell lymphomas of the germinal center type. In addition, large B-cell lymphomas in the CNS usually also express activated B-cell type markers, such as MUM-1, that are typically absent in systemic germinal B-cell lymphoma. ${ }^{7,8}$ These findings suggest that these tumors are biologically distinct from their systemic counterparts.

Molecular data have also revealed patterns of gene expression of CNS B-cell lymphomas that are distinct from those of systemic B-cell lymphoma. In one microarray study from our group, at least 100 genes were upregulated in PCNSL, relative to systemic disease. ${ }^{9}$ These included MYC and PIMI, proto-oncogenes that have also been shown to be mutated in PCNSL. ${ }^{10}$ Our data also suggested that interleukin-4 (IL-4) signaling may contribute to tumor progression. IL-4 was expressed both by PCNSL tumor cells and by the tumor vasculature. In addition, increased tumor or endothelial expression of STAT6, a downstream mediator of IL-4 signaling, predicted early progression and short survival in an independent set of patients all treated with high-dose methotrexate-based chemotherapy.

Comparison of two recent microarray studies of PCNSL, one conducted by our group ${ }^{11}$ and the other by Tun et al., ${ }^{12}$ revealed an overlapping set of genes that are differentially expressed in CNS lymphoma and systemic lymphomas (Table 1). A significant subset of these genes appear to constitute components of the extracellular matrix that are distinct in the brain, as opposed to nodal microenvironments (e.g., osteopontin; chitinase-3-like 1; collagen, type VI, alpha 1; and laminin, alpha 4). The differential upregulation of RGS13 in PCNSL is also notable, given that this protein may have a role in the modulation of responses to chemokines. ${ }^{13}$

Prior data also demonstrated distinct genetic alterations in PCNSL. For example, deletions of CDKN2A, a gene that produces the tumor suppressor protein $\mathrm{p} 14^{\mathrm{ARF}}$, were noted in $40 \%$ of PCNSL lesions. ${ }^{14}$ p14 ${ }^{\mathrm{ARF}}$, in turn, inhibits cell cycling by promoting p53 stabilization and
Table 1. Differential Gene Expression in Primary CNS Lymphoma

\begin{tabular}{ll}
\hline Gene Name & Symbol \\
\hline Upregulated & \\
Secreted phosphoprotein 1* & SPP1 \\
Complement component 1, q & $C 1 Q B$ \\
subcomponent, B chain & $H B A 2$ \\
Hemoglobin, alpha 2 & RGS13 \\
Regulator of G-protein signaling 13 & CHI3L1 \\
Chitinase 3-like 1 & TCL1A \\
T-cell leukemia/lymphoma 1A & \\
Downregulated & NNMT \\
Nicotinamide N-methyltransferase & VEGFC \\
Vascular endothelial growth factor C & COL6A1 \\
Collagen, type VI, alpha 1 & LXN \\
Latexin & LUM \\
Lumican & LAMA4 \\
Laminin, alpha 4 & \\
\hline
\end{tabular}

Adapted from Rubenstein et al. ${ }^{99}$ Gene symbols and names are updated according to the Human Gene Nomenclature Committee database (http://www.genenames.org; accessed May 2009).

*Previously known as osteopontin $(O P N)$ or bone sialoprotein 1 (BNSP).

inactivation of certain cyclin-dependent kinases. The importance of $\mathrm{p} 14^{\mathrm{ARF}}$ is supported by the finding that TP53 gene activation is detected in $20 \%$ to $40 \%$ of PCNSL tumors, but mutations of TP53 itself are rare. ${ }^{15}$ Alterations in the long arm of chromosome 12, which contains cell cycle genes MDM2 and CDK4, as well as the oncogene GLII, are also observed in PCNSL. ${ }^{16}$ Sixty-six percent of PCNSL lesions have deletions in the long arm of chromosome 6, which correlates with reduced expression of PTPRK, a candidate tumor suppressor protein, and, based on loss of heterozygosity analysis, 6q aberrations may predict short survival in PCNSL. ${ }^{17}$

One intriguing paradox in PCNSL is the question of how malignant $\mathrm{B}$-cells arise in or are introduced to the CNS. BCA-1 is a B-cell attracting chemokine, encoded by the $C X C L 13$ gene, that promotes B-cell homing to secondary lymphoid organs and that was recent shown to be widely expressed in PCNSL tumors. ${ }^{18}$ It appears that lymphoma cells within the brain, as well as the eye, have the capacity to respond to BCA-1 signals given their coexpression of CXCR5 $5{ }^{19}$ In addition, there is evidence that CNS lymphomas also express the chemokine SDF-1 (encoded by the CXCL12 gene) and its cognate receptor at high levels. ${ }^{15}$ Although it has also been hypothesized that systemic B-cells may express specific adhesion molecules that facilitate homing to the CNS, where these cells undergo transformation, no adhesion molecules specific to PCNSL have been identified to date. ${ }^{5}$

In AIDS patients, primary CNS lymphoma is strongly associated with Epstein-Barr virus (EBV), ${ }^{20,21}$ and EBV-associated PCNSL has also been reported in patients who are chronically exposed to immunosuppres- 
sive medications. ${ }^{22}$ In immunocompetent individuals, EBV infection may lead to the immortalization of otherwise normal B-cells. In AIDS, the proliferation of infected B-cells that would be suppressed by normal Tcells is left unchecked, and this is thought to allow proliferation and malignant transformation of the infected clone. ${ }^{23}$ EBV may also play a role in CNS trophism for PCNSL in the setting of AIDS, given that AIDS patients with EBV-positive systemic NHL have been shown to be more likely to have CNS involvement than patients whose tumors are EBV-negative. ${ }^{24}$ PCNSL is not associated with $\mathrm{EBV}$ in immunocompetent patients.

Recent proteomic analysis of CSF from CNS lymphoma patients identified approximately $80 \mathrm{CSF}$ proteins that were present in increased quantities in PCNSL, relative to controls. ${ }^{25}$ Increased levels of one of these proteins, antithrombin III (a serine protease inhibitor best known for its role in the regulation of blood clotting), may be associated with poor overall survival in patients with relapsed disease.

\section{EPIDEMIOLOGY AND RISK FACTORS}

The best-established risk factor for primary CNS lymphoma is immunodeficiency. The importance of this risk factor was borne out by a 1996 retrospective study that demonstrated a several thousand-fold increased incidence of CNS lymphoma in patients with AIDS, compared with the general population ${ }^{3}$; AIDS patients had an approximately $20 \%$ lifetime risk of developing the disease. The advent of highly active antiretroviral therapy, which led to immune recovery in many AIDS patients, was associated with a decreased incidence of primary CNS lymphoma, as demonstrated in a 2005 study that found a marked decrease in the incidence of primary CNS lymphoma from 8.6 per 1000 patients between 1988 and 1995 to 1.1 per 1000 patients between 1996 and $2000 .{ }^{4}$ After the peak of the AIDS epidemic, a net decrease in the overall incidence of primary CNS lymphoma was noted in a study based on a systematic review of the Surveillance, Epidemiology, and End Results (SEER) database. ${ }^{26}$ Although this review did not separate primary CNS lymphoma patients with and without comorbid human immunodeficiency virus (HIV) infections, the decreasing incidence of primary CNS lymphoma was not observed in women who had a substantially lower incidence of HIV than men at the peak of the HIV epidemic in the 1980s. Primary CNS lymphoma has also been observed in other settings of immunodeficiency, such as in patients with rheumatologic conditions $^{22,27}$ and in transplant recipients ${ }^{27,28}$ who are managed chronically with immunosuppressive medications.

Several authors have suggested that there has been a dramatic increase in the incidence of primary CNS lym- phoma in immunocompetent patients. Two studies based on the SEER database demonstrated a net increase in the incidence of primary CNS lymphoma between the 1970s and the 1990s in a population that excluded never-married persons or persons of unknown marital status. ${ }^{29}$ This strategy was presumed to deplete the study population of homosexual men, who comprised a substantial majority of HIV-infected persons during the time period of the study. Another group linked AIDS and cancer registries in a number of states to identify primary CNS lymphoma patient with and without concurrent AIDS diagnoses. They found that the incidence of non-AIDS patients with CNS lymphoma increased seven-fold between 1982 and $1989 .^{3}$

Primary CNS lymphoma can affect both sexes over a wide age range, although there are notable demographic differences between immunocompetent and immunocompromised individuals. In patients with no detectable immune deficiency, the male-to-female ratio is $3: 2$, and the mean age at diagnosis is the fifth decade. ${ }^{27}$ In contrast, perhaps due in major part to the AIDS epidemic, the male-to-female ratio in immunocompromised patients is 10:1, and the mean age at diagnosis is the third decade.

\section{CLINICAL FEATURES}

The onset of symptoms in PCNSL tends to be insidious, and clinical presentation depends to a substantial extent on the location of the disease. Immunocompetent patients are more likely to present with a single mass lesion on the cerebrum, ${ }^{30}$ but nearly all patients with AIDS present with multifocal disease. Immunocompetent patients present less commonly with tumors in the basal ganglia and cerebellum. Patients with PCNSL tumors in the cerebrum may present with focal neurological deficits, manifestations of increased intracranial pressure, cognitive deficits, or neuropsychiatric changes. Focal neurological deficits are noted in more than one half of patients, and may include aphasia, motor, or visual deficits. Signs and symptoms of increased intracranial pressure are seen in approximately one third of patients, and may include headaches, nausea, vomiting, papilledema, and lethargy. Cognitive, behavioral, mood, and personality changes are also seen in approximately one third of patients, often in the setting of frontal lobe, callosal, or white matter lesions. These symptoms may include depression, changes in executive function, memory impairment, and confusion. Approximately $11 \%$ of all patients present with seizures.

Sites of disease outside the brain parenchyma lead to unique constellations of symptoms. Patient with leptomeningeal lymphoma, with or without mass lesions in the brain parenchyma, may develop meningismus, cranial nerve palsies, headaches, or signs and symptoms of 
hydrocephalus and increased intracranial pressure. Approximately $10 \%$ to $20 \%$ of patients present with intraocular lymphoma. Patients with isolated ocular disease may present with an insidious decrease in visual acuity; because this symptom is nonspecific, there can be a long time lag between the onset of symptoms and diagnosis. ${ }^{30,31}$ Patients may also note vitreal opacities that increase over time, ${ }^{32}$ and visual hallucinations have been described. Spinal lesions are uncommon in PCNSL, and they typically manifest as discrete intramedullary nodules. $^{30}$ These may be associated with motor or sensory levels similar to other mass lesions affecting the spinal cord.

PCNSL is diagnosed in patients over a large range of ages. The peak incidence differs markedly between immunocompetent patients, with a median age at diagnosis in the sixth decade, and immunocompromised patients, with a median age at diagnosis of 30 years.

\section{DIAGNOSIS}

The vast majority of patients with PCNSL present with a mass lesion in brain parenchyma. Definitive diagnosis typically involves high-resolution imaging of the brain, exclusion of systemic disease through additional imaging and staging procedures, and the pathologic analysis of tumor specimens most commonly obtained via stereotactic surgical brain biopsy. Diagnosis may also be established by CSF analysis using either cytology or flow-cytometry, and pathologic analysis of vitreal biopsies is also useful to establish the diagnosis of intraocular lymphoma.

\section{Imaging}

Gadolinium contrast-enhanced MRI is the imaging modality of choice for evaluation of PCNSL. The most common finding on MRI for immunocompetent patients with PCNSL is a contrast-enhancing solitary tumor located in one of the cerebral hemispheres. ${ }^{2,33}$ For example, in one series, $55 \%$ of patients had lesions in the cerebral hemispheres, $27.5 \%$ had lesions in the basal ganglia, and $27.5 \%$ had lesions in the corpus callosum. Leptomeningeal involvement was found in $12.5 \%$, but nearly all $(97.5 \%)$ had lesions adjacent to the subarachnoid space. ${ }^{33}$ Only one fourth of patients with normal immune systems have more than one lesion, whereas more than one half of AIDS patients have multifocal disease. $^{2}$

The pattern of contrast enhancement is uniform in 55\% to $60 \%$ of cases, ${ }^{2}$ but $\mathrm{T}_{2}$ signal intensity may be heterogeneous $(55 \%)$, uniformly hyperintense $(15 \%)$, or uniformly hypointense $(32.5 \%) .{ }^{33}$ Moderate to extensive vasogenic edema surrounding PCNSL lesions has been described in the majority of cases, but significant peritumoral edema is observed less frequently in PCNSL than in gliomas and in solid tumor brain metastases. ${ }^{2}$ Necrosis is more common in patients previously treated with corticosteroids. Positron emission tomography (PET) is thought to be of limited utility in the diagnosis of PCNSL, due to the high background metabolic activity of the brain.

Newer imaging techniques may confer additional diagnostic and even prognostic information. Histologically, PCNSL often exhibits an angiocentric growth pattern. Although conventional MRI findings in PCNSL may be similar to those of other tumors, there is evidence that perfusion MRI may be able to exploit the this unique growth pattern to differentiate PCNSL lesions from other tumors, such as glioblastoma multiforme tumors, or infectious lesions, such as those seen in toxoplasmosis. ${ }^{34}$

Other investigators seek to exploit differences in a tumor's cellular density to determine tumor grade based on imaging alone. For example, the apparent diffusion coefficient (ADC), a measure of the diffusion of water molecules in cerebral tissue, has been correlated with cellular density in human subjects with PCNSL and astrocytoma; ADC values were significantly different between the two groups. ${ }^{35}$ Changes in ADC are correlated with treatment associated changes in tumor cell density, proliferation, and therapeutic response in murine models of NHL. ${ }^{36}$ Increased cellular density has been inversely correlated with overall survival in PCNSL, ${ }^{9}$ and recent data suggest that pretherapeutic ADC, as a possible surrogate marker for cellular density, predicts clinical outcome in PCNSL patients treated with methotrexatebased chemotherapy regimens.

\section{Biopsy and diagnosis}

Although surgical biopsy is usually required to diagnose PCNSL, under special circumstances it may be possible to diagnose PCNSL through imaging and CSF examination. The CSF cytology is diagnostic in a minority of patients, with estimates ranging from $15 \%$ to $31 \%,{ }^{37-39}$ although it has been suggested that repeat CSF cytological examinations may facilitate diagnosis. ${ }^{37}$ Magnetic resonance imaging of brain and spine, with and without gadolinium, is useful for complete staging of the neuroaxis.

A recent proteomic study of CSF in PCNSL patients revealed a distinct protein profile in association with the disease, and it has been suggested that a panel of CSF protein biomarkers proteins including antithrombin III may ultimately facilitate noninvasive diagnosis as well as risk stratification. ${ }^{25}$

In the setting of AIDS, there is a close association between PCNSL and infection with the Epstein-Barr Virus (EBV), and EBV PCR has a high positive predictive value for the disease. ${ }^{20}$ Because single photon emission computed tomography (SPECT) and PET imaging ${ }^{40}$ can distinguish PCNSL from infectious causes such as cere- 
bral toxoplasmosis in AIDS patients, it has been suggested that patients with positive EBV results from polymerase chain reaction and findings on PET or SPECT that are consistent with PCNSL do not require brain biopsies. Of note, stereotactic brain biopsy is associated with up to a $4 \%$ risk of significant intracranial hemorrhage $^{41}$ and biopsy is nondiagnostic in $8 \%$ to $9 \%$ of patients with intra-axial mass lesions. ${ }^{42,43}$ In addition, because corticosteroids induce transient treatment responses in PCNSL, ${ }^{44}$ prior exposure to systemic corticosteroids is expected to decrease the diagnostic yield of stereotactic biopsy.

\section{Differential diagnosis and staging}

The vast majority (90\%) of patients with lymphomatous involvement of the CNS will not have systemic disease. Nonetheless, a thorough assessment for systemic disease is crucial, because of the obvious treatment implications. Patients with both systemic and CNS disease should be assigned a diagnosis of stage IV NHL with CNS involvement. The National Clinical Cancer Network $^{45}$ recommends CT imaging of the chest, abdomen, and pelvis, with consideration of PET imaging. Additional tests for systemic disease include a bone marrow biopsy, and there is recent evidence suggesting that identification of clonally rearranged immunoglobulin-heavy chain (IgH) genes points to subclinical bone marrow involvement. ${ }^{46} \mathrm{~A}$ testicular ultrasound should also be considered, given that approximately $30 \%$ of testicular lymphomas metastasize to the brain. A complete blood count with a platelet count and white cell differential is indicated as are liver function tests. It is also important to screen patients for the human immunodeficiency virus (HIV), given the association between PCNSL and HIV, as well as the treatment implications of this comorbidity.

Serum lactate dehydrogenase (LDH) levels should be assessed, because elevation of serum LDH is associated with worse overall prognosis. ${ }^{47}$ Of patients with lymphoma in the brain, $10 \%$ to $20 \%$ also have intraocular disease, and a slit lamp examination is recommended. PCNSL tumors are avascular on angiography, and their tendency to develop adjacent to ventricles and cortical convexities suggests dissemination via the CSF; $10 \%$ to $20 \%$ of patients with PCNSL present with overt leptomeningeal lymphoma. Thus, all patients should undergo a lumbar puncture, unless increased intracranial pressure prohibits this procedure. Key clinical prognostic markers include Eastern Cooperative Oncology Group performance status $(<1)$ and age greater than 60 years.

\section{TREATMENT}

Left untreated, the median life expectancy for a patient with newly diagnosed PCNSL is 3 months. PCNSL will, however, respond to a number of therapeutic interven- tions, including systemic corticosteroid therapy, external beam radiation, chemotherapy, and immunotherapy. With the advent of new therapies, 5-year overall survival rates of up to $30 \%$ to $40 \%$ have been reported. ${ }^{48}$ Indeed, the past two decades have witnessed the development of a variety of novel therapeutic strategies based upon the use of chemotherapy that may lead to durable remissions with reasonable toxicities and which may obviate the neurotoxicity of WBRT.

\section{Clinical stabilization}

Because mass lesions in the brain and spinal cord can have devastating consequences, patients with PCNSL may require timely medical or surgical decompression even before they are formally diagnosed. PCNSL lesions are sensitive to corticosteroids, which kill tumor cells and decrease tumor-associated edema; response rates as high as $70 \%$ have been reported. ${ }^{44}$ Responses to corticosteroids are transient, however, and corticosteroids can decrease the diagnostic yield of surgical biopsies, thus delaying diagnosis and definitive therapies. It is therefore recommended that, if medically feasible, surgical biopsies be obtained prior to the initiation of corticosteroid therapy. Surgical intervention may also be required when PCNSL mass lesions lead to increased intracranial pressure, actual or impending herniation, or other neurosurgical emergencies, and a surgical biopsy is often required for diagnosis. Nonetheless, surgical resection or debulking do not confer survival benefits, and reported median overall survival durations with surgery alone ${ }^{38,49-51}$ are similar to overall survival durations in untreated patients.

\section{First-line therapy}

Radiation therapy. In the past, external beam WBRT, without systemic chemotherapy, was the mainstay of definitive treatment for PCNSL. External beam radiation yields overall response rates in PCNSL of $90 \%$ with complete response rates of approximately $60 \%$. Median overall survival in PCNSL patients treated with external beam radiation alone has been estimated at 12 to 18 months. ${ }^{52}$ Based on the infiltrative and multifocal nature of PCNSL, whole-brain irradiation has been the preferred radiation modality. There is no evidence that irradiating the spine is advantageous in the absence of detectable disease in the spine, and the majority of patients with intracranial disease relapse in the irradiated field, ${ }^{38,53}$ suggesting that treatment failures stem from inherent limitations of irradiation rather than from inadequate treatment fields.

In addition to limitations in efficacy, WBRT frequently leads to neurocognitive deficits. Up to two thirds of patients who were treated with whole-brain irradiation experienced delayed neurotoxicity, which may include cerebellar dysfunction, progressive dementia, and urinary incontinence. ${ }^{48,54}$ Lower doses of WBRT appear to be associated with decreased antitumor efficacy. For ex- 
ample, one group found that decreasing the dose from 45 Gy to 30.6 Gy decreased both progression-free survival and overall survival. ${ }^{55}$

Chemoradiation. To increase response and survival rates in PCNSL lymphoma, a number of treatment regimens have developed that combine WBRT with systemic chemotherapy, but treatment with these regimens is also associated with high rates of treatment-related neurotoxicity. By the 1980s, combined modality regimens as first-line therapy showed promise of improved overall survival. One regimen combined induction chemotherapy with systemic and intrathecal methotrexate, followed by cranial irradiation and then two cycles of systemic cytarabine in a non-AIDS population with PCNSL. ${ }^{56}$ The median overall survival was 42.5 months, substantially longer than historical overall survival estimates seen in patients treated with radiation alone. The same group subsequently published a number of studies on the long-term adverse neurological effects of treatment with combined modality therapy. In one retrospective, a $24 \%$ incidence of neurotoxicity with increasing incidence noted over time. In this study, the medical records of patients with primary CNS lymphoma were reviewed for documentation of neurologic deterioration that could not be attributed to tumor progression or to other causes, and it was noted that many patients died before any delayed neurotoxicity, if present, would be expected. ${ }^{57}$ A European study of PCNSL patient treated with methotrexate and WBRT found a much higher rate of cognitive impairment, with impairment in 63\% of patients treated with methotrexate and WBRT, despite a complete tumor response. ${ }^{54}$

More aggressive chemotherapy regimens have subsequently been used in conjunction with WBRT, in attempts to increase survival and by decreasing WBRT doses to reduce toxicity. Another regimen evaluated by the Radiation Therapy Oncology Group (RTOG) combined induction chemotherapy using five cycles of highdose methotrexate with vincristine, procarbazine, and intrathecal methotrexate followed by WBRT with a total dose of $45 \mathrm{~Gy} .{ }^{58}$ In this study, $36 \%$ of patients had a complete response after induction chemotherapy, and $94 \%$ responded overall. The median progression-free survival with this regimen was 24 months, and the overall survival was 36.9 months $(n=102)$. The authors noted a significantly longer overall survival in patients under 60 years of age (median overall survival, 50.4 months), and a $15 \%$ rate of delayed neurological toxicity, which was often fatal. ${ }^{58}$

In a later study by the same group, patients were treated with five to seven cycles of rituximab, methotrexate, procarbazine, and vincristine (R-MPV), followed by reduced-dose WBRT of 23.4 Gy in patients achieving a complete response to chemotherapy and by standard dose WBRT of 45 Gy in all others. ${ }^{59}$ Patients were then treated with two cycles of high-dose cytarabine. The overall response rate in the study was $93 \%$, with $78 \%$ complete response after chemotherapy. The 2-year overall survival rates in patients with and without a complete response after induction chemotherapy were $67 \%$ and $57 \%$, respectively. A small prospective case series of the patients treated with the lower dose (23.4 Gy) of WBRT showed improving executive function over the 2-year study period, with persistent deficits overall in verbal memory and motor speed at 2 years. ${ }^{60}$

Other chemoradiation regimens have included an intensive induction regimen consisting of a combination of systemic and intrathecal methotrexate, teniposide, carmustine, methylprednisolone, and cytarabine followed by 40 Gy of WBRT. This regimen yielded an overall response rate of $81 \%$ and a median overall survival of 46 months, at the expense of a $10 \%$ treatment-related mortality rate. ${ }^{61}$

Chemotherapy Alone. The use of cytotoxic chemotherapy alone in PCNSL has been studied for more than 30 years and the findings of these studies highlight the unique biology and treatment challenges surrounding PCNSL. As early as the mid 1970s, case reports described patients who were successfully treated with methotrexate after unsuccessful treatment with WBRT. ${ }^{62,63}$ Although PCNSL shares many pathological characteristics with systemic NHL, responses may differ markedly between systemic and CNS lymphoma to a given chemotherapeutic agent. For example, the combination of systemic cyclophosphamide, doxorubicin, vincristine and corticosteroids can be highly effective in systemic large B-cell lymphoma, but is ineffective in PCNSL, adding no survival benefit when combined with WBRT. ${ }^{64,65}$ This has been attributed to the poor penetration of many chemotherapeutic agents into the CNS due to the blood-brain barrier, although there are also data suggesting that molecular differences exist between PCNSL and its systemic counterparts. ${ }^{11}$

Among pharmacologically active agents, distribution of a drug and its pharmacokinetics is of particular important. This is certainly true of methotrexate, which forms the backbone of most systemic cytotoxic therapy regimens for PCNSL. In patients with meningeal leukemia or carcinomatosis, CNS methotrexate levels have been shown to be one thirtieth of systemic levels. ${ }^{66} \mathrm{Sev}$ eral approaches to overcoming this obstacle have been attempted, including the administration of intrathecal methotrexate and the use of high-dose systemic methotrexate. Overall, pharmacokinetic and clinical data favor the superiority of high-dose systemic therapy. For example, one study of patients with solid-tumor neoplastic meningitis demonstrated that, compared with intrathecal methotrexate, high-dose systemic methotrexate therapy yielded much more sustained cytotoxic drug concentrations in the CSF. ${ }^{67}$ Retrospective studies have also dem- 
onstrated that there is no additional benefit derived from administering intrathecal methotrexate to patients with PCNSL who have received adequate doses of systemic, high-dose methotrexate. ${ }^{68}$

Another approach for attaining adequate CSF chemotherapy concentrations in PCNSL has been to alter the permeability of the blood-brain barrier using intra-arterial or intravenous osmotic agents. In one study, 53 patients with PCNSL were treated with mannitol followed by high-dose intra-arterial methotrexate, cyclophosphamide, and etoposide. ${ }^{69}$ The overall and complete response rates were 86 and $71 \%$, respectively. Another study from the same group examining the efficacy of disruption of the tight junctions of the CNS endothelium with mannitol, but with oral procarbazine substituted for parenteral etoposide in some patients, yielded a complete response rate of $65 \%$, an estimated 5-year survival rate of $42 \%$, and a median survival of 40.7 months. $^{70}$ Acute adverse effects in theses studies included seizures, strokes, and venous thrombolic disease, but there was no evidence of treatment-related neuropsychological deficits in patients who had not been exposed to WBRT.

In the $1990 \mathrm{~s}$, more formal evaluation of methotrexatebased chemotherapy regimens without WBRT suggested that these regimens could be highly effective with a substantially better side effect profile than WBRT containing regimens. One case series, expanding on data from a small cohort of patients who refused WBRT, demonstrated durable treatment responses in the majority of patients, with little or no significant treatment-associated toxicity. ${ }^{71}$ Another group based at the Memorial Sloan-Kettering Cancer Center in New York examined methotrexate-based chemotherapy regimens in 13 elderly patients (median age, 74 years) and found responses in 12 of 13. Performance scores improved in 11 of 13 patients, cognitive function improved in 8 of 9 patients with pretreatment cognitive deficits, and only 1 patient developed new cognitive deficits in the setting of progressive disease and possible methotrexate-induced leukoencephalopathy.

A 1999 consortium study led by Fred Hochberg's group at Massachusetts General Hospital examined the efficacy, toxicity, and quality of life in 31 immunocompetent PCNSL patients who were treated with high-dose methotrexate alone. ${ }^{72}$ Standard treatment dosing for methotrexate was $8 \mathrm{~g} / \mathrm{m}^{2}$ every 2 weeks, with dose reductions for suboptimal renal function. All patients responded to treatment, and $65 \%$ of patients had a complete response. Performance status also improved dramatically with treatment. The median Karnofsky Performance Scale score improved from 40 to 90 with treatment. The median overall survival was more than 30 months, and $90 \%$ of patients who had a complete response to therapy were alive at 2 years. Treatment toxicities were infrequent and included leucopenia, nonoli- guric acute renal failure, and mucositis. A cohort of 11 patients who achieved a complete response to chemotherapy was monitored for 2 years with cognitive and quality of life assessments and all had preserved cognitive function and memory.

A subsequent, multicenter trial of intensive high-dose methotrexate conducted by the New Approaches to Brain Tumor Therapy CNS Consortium demonstrated a 52\% complete response rate and a $74 \%$ overall response rate in 25 patients studied. ${ }^{73}$ Median progression-free survival was 12.8 months, and median overall survival had not been reached at more than 22 months. Again, no delayed neurotoxicity was observed. One European trial of intensive, high-dose methotrexate for PCNSL yielded more modest benefits, with only a $30 \%$ complete response rate in the 37 patients and refractory disease in $38 \%$ of them. ${ }^{74}$ Consistent with prior results, however, delayed neurotoxicity rates in surviving patients were much lower $(10 \%)$ in patients who were never treated with WBRT salvage therapy, compared with those who were $(58 \%)$.

Given the favorable toxicity profile of high-dose methotrexate-based chemotherapy regimens, new regimens are being developed that integrate high-dose methotrexate and additional agents. Our group initiated a trial of the combination of high-dose methotrexate, temozolomide, and rituximab (MTR) followed by consolidation with cytarabine and etoposide, a regimen currently under study nationally as CALGB trial 50202 .

Temozolomide is a congener of dacarbazine that is taken orally; it has excellent bioavailability in the CSF and a favorable toxicity profile. The agent is approved for use in malignant gliomas, and it has demonstrated efficacy as monotherapy in PCNSL ${ }^{75,76}$ as salvage therapy in patients previously treated with high-dose methotrexate; recent data for 36 patients suggest a $31 \%$ overall response rate and $31 \%$ 1-year survival.

Rituximab is a monoclonal antibody directed toward CD20, a cell-surface protein expressed exclusively on mature B-cells and not on neurons or glia. The efficacy of rituximab in systemic B-cell lymphoma is now well established based on clinical trials, including a large randomized trial in Europe in which complete response rates to CHOP chemotherapy (cyclophosphamide-doxorubicin-vincristine-prednisone) increased from 63 to $76 \%$ with the addition of rituximab to the therapeutic regimen. ${ }^{77}$ In addition, patients treated with $\mathrm{R}-\mathrm{CHOP}$ (rituximab-CHOP) had fewer relapses and longer overall survival. A major problem with using rituximab for primary CNS lymphoma has been poor bioavailability of the drug in the CNS, with CSF levels of the drug approximately $0.1 \%$ of the serum levels that are achieved. ${ }^{78}$ However, it has been suggested that higher CSF levels may be attained in the setting of leptomeningeal inflammation, as suggested by recent data showing that CSF/ 
serum rituximab ratios correlated with $\mathrm{CSF} /$ serum albumin ratios in patients with neurological autoimmune disorders. Similar changes have been seen after WBRT in CSF concentrations of systemically administered trastuzumab, ${ }^{79}$ a monoclonal antibody directed against the cell surface antigen HER2 that is overexpressed in many breast cancer patients.

Preliminary data from a pilot trial at the University of California, San Francisco to evaluate the MTR regimen of the CALGB 50202 trial showed a $56.5 \%$ complete response rate. The median progression-free and overall survival had not been reached at 33 months. The regimen was generally well tolerated, with no treatment-related mortality, and no patients developed significant treatment-related neurotoxicity. ${ }^{80}$

Another aggressive chemotherapy regimen developed in Bonn, Germany, involving high-dose systemic methotrexate, intrathecal methotrexate, ifosfamide, cyclophosphamide, cytarabine, prednisone, and vinca alkaloids yielded a $71 \%$ overall response rate, with $61 \%$ complete response in 65 patients. The median progression-free and overall survival times were 21 and 50 months, respectively; however, $9 \%$ of patients died from treatment-related complications, and 19\% suffered from Ommaya reservoir infections. ${ }^{81} \mathrm{~A}$ subsequent study by the same group examined a similar regimen without intraventricular methotrexate in an effort to circumvent Ommaya reservoir-related complications. ${ }^{82}$ They detected a high rate of early relapses, with a mean response duration of only 10 months in responding patients. Based on their experience, these investigators concluded that intraventricular therapy is an essential part of the therapeutic regimen, in contrast to earlier retrospective studies suggesting no benefit from administering intrathecal methotrexate to PCNSL patients receiving high-dose methotrexate systemically. ${ }^{68}$

Stem-cell transplantation. Autologous stem cell transplantation, a therapy that has shown efficacy in relapsed and high-risk systemic lymphoma, has also been used in PCNSL. Several different regimens have been examined in the first-line setting. Abrey et al. ${ }^{83}$ treated 28 patients with induction chemotherapy with methotrexate at $3.5 \mathrm{~g} / \mathrm{m}^{2}$ and cytarabine at $3 \mathrm{~g} / \mathrm{m}^{2}$ for 2 days, conditioning with carmustine, etoposide, cytarabine, and melphalan, and then autologous stem-cell rescue. The objective response rate with this regimen was a modest $57 \%$, and median event-free survival was only 9.3 months; there was one treatment-related death.

Several groups have examined salvage and transplantation regimens in the first-line setting that call for WBRT for patients with incomplete treatment responses. One recent example is a 2008 study that examined tandem high-dose methotrexate induction followed by conditioning with busulfan and thiotepa and autologous stem-cell rescue. ${ }^{84}$ Patients without a response to induc- tion or without a complete remission after transplantation received WBRT. Of the 23 patients treated, 3 died during therapy and 3 died of delayed neurotoxicity after WBRT. The 2-year overall survival rate for all patients was $48 \%$; for transplanted patients, it was $61 \%$.

\section{Salvage regimens}

Chemotherapy, radiation, and transplantation. Soussain et al. ${ }^{85}$ have had success treating refractory or relapsed PCNSL with intensive salvage chemotherapy followed by autologous stem-cell transplantation. Their regimen includes two cycles of high-dose cytarabine and etoposide, with chemosensitive patients subsequently receiving conditioning with thiotepa, busulfan, and cyclophosphamide followed by autologous stem-cell rescue. Forty-three patients were enrolled in the study, and 27 proceeded to conditioning and transplantation. Of those 27 patients, 26 entered complete remission. The median overall survival was 18.3 months in all patients and 58.6 months in patients who completed intensive chemotherapy with stem cell rescue. Three patients died from treatment-related toxicity after salvage therapy, two died from sepsis, one died from CNS toxicity, and one died from hemorrhage after a brain biopsy that followed salvage chemotherapy. This regimen does not appear to be a viable alternative for patients older than 60 years; in an earlier study, five of seven patients over 60 years of age died of treatment-related causes.

Other approaches to salvage therapy for relapse or refractory PCNSL have included retreatment with methotrexate, WBRT, and combination chemotherapy. In a study at Massachusetts General Hospital, retreatment with high-dose methotrexate yielded a high response rate, durable remissions, and acceptable levels of toxicity. ${ }^{86}$ Of 22 patients (median age, 58 years) receiving high-dose methotrexate after a first relapse, 20 achieved a complete remission. The median overall survival in this group was 61.9 months. Combination chemotherapy approaches have included procarbazine, lomustine, and vincristine, ${ }^{87}$ which yielded an $86 \%$ overall response rate in seven patients, four of whom remained disease-free at 1 year after relapse. Salvage therapy with temozolomide ${ }^{75,76}$ yielded a $31 \%$ overall response rate and 1 -year survival of $31 \%$ in 36 patients. In patients for whom initial induction with high-dose methotrexate failed, WBRT with a median dose of 36 Gy yielded an overall response rate of $74 \%$ and a median overall survival of 10.9 months. ${ }^{88}$ Stereotactic radiosurgery was examined in a small series of nine patients: five had recurrences distant to the site of radioablation; four survived for 1 year. $^{89}$

Immunotherapy. Immunotherapy directed toward the CNS is a promising approach to treating patients with relapsed or refractory disease, an approach that may also provide insights into the mechanisms of immune re- 
sponse and resistance in PCNSL. As already noted, rituximab is a monoclonal antibody directed toward CD20, a cell surface protein expressed on mature B-cells but not neurons or glia. Rituximab has well-documented efficacy in systemic B-cell lymphoma, and rituximab-containing regimens have become the standard of care in this setting. However, systemically administered rituximab yields poor bioavailability in the CSF, with levels estimated at $0.1 \%$ of those measured in the serum. ${ }^{78}$ In a phase I study of intrathecal rituximab in nine patients with relapsed PCNSL, four achieved complete cytological responses and two achieved partial responses. ${ }^{90}$ Dose-limiting toxicities included grade 3 hypertension observed in two patients treated at a 50-mg dose level. In addition, the combination of intrathecal rituximab plus liposomal cytarabine has recently been evaluated in 14 patients with recurrent lymphomatous meningitis. Preliminary evidence suggests that this combination has no additive toxicity and is associated with moderate activity in this setting. ${ }^{91}$

Intraocular lymphoma. Many of the therapeutic modalities applied for in intraocular lymphoma parallel those used in PCNSL that is restricted to the brain. Twenty-four-hour continuous intravenous administration of high-dose methotrexate yields cytotoxic levels in the anterior chamber of the eye within 7 hours, ${ }^{92}$ and both methotrexate and cytarabine have activity in intraocular lymphoma. ${ }^{93,94}$ In a study of systemic methotrexate for intraocular lymphoma, seven of nine patients had objective responses, and four of these responses were sustained after 8 to 36 months of follow-up. Ocular radiation is also effective ${ }^{31}$ but, as in PCNSL in the brain, treatmentassociated morbidity is common, including cataracts, retinal damage, and vision loss. Furthermore, sustained responses to ocular radiation, systemic chemotherapy, and the two modalities combined are seen in only a minority of patients with intraocular lymphoma. ${ }^{31}$

Serial intravitreal injections of methotrexate led to clinical remissions in nearly all of 36 patients treated in one series, and none of these patients were noted to have had an intraocular recurrence..$^{95}$ Intravitreal injections of rituximab yields concentrations of this antibody of greater than $10 \mathrm{ng} / \mathrm{mL}$ for 72 days, ${ }^{96,97}$ and anecdotal evidence suggests that the drug may be effective in this setting. For example, one group used rituximab alone in two patients who could no longer tolerate intravitreal methotrexate; both patients had objective responses. ${ }^{98}$

\section{NEW APPROACHES AND FUTURE DIRECTIONS}

Given the evidence for neurotoxicity associated with standard genotoxic agents in primary CNS lymphoma, in particular with WBRT, there is considerable interest in the evaluation of new genotoxic and targeted strategies to treat this disease. Approaches under consideration include the analysis of pemetrexed disodium, a recently approved folate antagonist, and the up-front use of rituximab in combination with the standard methotrexate, procarbazine, and vincristine, as well as ongoing evaluation of blood-brain barrier disruption.

\section{REFERENCES}

1. Hochberg FH, Baehring JM, Hochberg EP. Primary CNS lymphoma. Nat Clin Pract Neurol 2007;3:24-35.

2. Fine HA, Mayer RJ. Primary central nervous system lymphoma. Ann Intern Med 1993;119:1093-1104.

3. Cote TR, Manns A, Hardy CR, Yellin FJ, Hartge P; AIDS/Cancer Study Group. Epidemiology of brain lymphoma among people with or without acquired immunodeficiency syndrome. J Natl Cancer Inst 1996;88:675-679.

4. Diamond C, Taylor TH, Aboumrad T, Anton-Culver H. Changes in acquired immunodeficiency syndrome-related non-Hodgkin lymphoma in the era of highly active antiretroviral therapy: incidence, presentation, treatment, and survival. Cancer 2006;106:128-135.

5. Kadoch C, Treseler P, Rubenstein JL. Molecular pathogenesis of primary central nervous system lymphoma. Neurosurg Focus 2006;21(5):E1.

6. Montesinos-Rongen M, Kuppers R, Schluter D, et al. Primary central nervous system lymphomas are derived from germinalcenter B cells and show a preferential usage of the V4-34 gene segment. Am J Pathol 1999;155:2077-2086.

7. Camilleri-Broët $S$, Crinière $E$, Broët $P$, et al. A uniform activated B-cell-like immunophenotype might explain the poor prognosis of primary central nervous system lymphomas: analysis of 83 cases. Blood 2006;107:190-196.

8. Lin CH, Kuo KT, Chuang SS, et al. Comparison of the expression and prognostic significance of differentiation markers between diffuse large B-cell lymphoma of central nervous system origin and peripheral nodal origin. Clin Cancer Res 2006;12:1152-1156.

9. Rubenstein JL, Fridlyand J, Shen A, et al. Gene expression and angiotropism in primary CNS lymphoma. Blood 2006;107:37163723 .

10. Montesinos-Rongen M, Van Roost D, Schaller C, Wiestler OD, Deckert M. Primary diffuse large B-cell lymphomas of the central nervous system are targeted by aberrant somatic hypermutation. Blood 2004;103:1869-1875.

11. Rubenstein JL, Shen A, Fridlyand J, et al. Gene expression profile analysis of primary CNS lymphoma: class distinction and outcome prediction. Proc Am Assoc Cancer Res 2004;45:abstract 4433.

12. Tun HW, Personett D, Baskerville KA, et al. Pathway analysis of primary central nervous system lymphoma. Blood 2008;111: 3200-3210.

13. Han JI, Huang NN, Kim DU, Kehrl JH. RGS1 and RGS13 mRNA silencing in a human B lymphoma line enhances responsiveness to chemoattractants and impairs desensitization. J Leukoc Biol 2006; 79:1357-1368.

14. Cobbers JM, Wolter M, Reifenberger J, et al. Frequent inactivation of $C D K N 2 A$ and rare mutation of TP53 in PCNSL. Brain Pathol 1998;8:263-276.

15. Rubenstein JL, Treseler P, O'Brien JM. Pathology and genetics of primary central nervous system and intraocular lymphoma. Hematol Oncol Clin North Am 2005;19:705-717, vii.

16. Harada K, Nishizaki T, Kubota H, et al. Distinct primary central nervous system lymphoma defined by comparative genomic hybridization and laser scanning cytometry. Cancer Genet Cytogenet 2001;125:147-150.

17. Nakamura M, Kishi M, Sakaki T, et al. Novel tumor suppressor loci on 6q22-23 in primary central nervous system lymphomas. Cancer Res 2003;63:737-741.

18. Mazzucchelli L, Blaser A, Kappeler A, et al. BCA-1 is highly expressed in Helicobacter pylori-induced mucosa-associated lymphoid tissue and gastric lymphoma. J Clin Invest 1999;104:R49R54. 
19. Smith JR, Braziel RM, Paoletti S, et al. Expression of B-cellattracting chemokine 1 (CXCL13) by malignant lymphocytes and vascular endothelium in primary central nervous system lymphoma. Blood 2003;101:815-821.

20. Antinori A, De Rossi G, Ammassari A, et al. Value of combined approach with thallium-201 single-photon emission computed tomography and Epstein-Barr virus DNA polymerase chain reaction in CSF for the diagnosis of AIDS-related primary CNS lymphoma. J Clin Oncol 1999;17:554-560.

21. Bossolasco S, Cinque P, Ponzoni M, et al. Epstein-Barr virus DNA load in cerebrospinal fluid and plasma of patients with AIDSrelated lymphoma. J Neurovirol 2002;8:432-438.

22. Kleinschmidt-DeMasters BK, Damek DM, Lillehei KO, Dogan A, Giannini C. Epstein Barr virus-associated primary CNS lymphomas in elderly patients on immunosuppressive medications. J Neuropathol Exp Neurol 2008;67:1103-1111.

23. Penn I. The role of immunosuppression in lymphoma formation. Springer Semin Immunopathol 1998;20:343-355.

24. Cingolani A, Gastaldi R, Fassone L, et al. Epstein-Barr virus infection is predictive of CNS involvement in systemic AIDSrelated non-Hodgkin's lymphomas. J Clin Oncol 2000;18:33253330.

25. Roy S, Josephson SA, Fridlyand J, et al. Protein biomarker identification in the CSF of patients with CNS lymphoma. J Clin Oncol 2008:26:96-105.

26. Kadan-Lottick NS, Skluzacek MC, Gurney JG. Decreasing incidence rates of primary central nervous system lymphoma. Cancer 2002;95:193-202.

27. Schabet M. Epidemiology of primary CNS lymphoma. J Neurooncol 1999;43:199-201.

28. Penn I, Porat G. Central nervous system lymphomas in organ allograft recipients. Transplantation 1995;59:240-244.

29. Olson JE, Janney CA, Rao RD, et al. The continuing increase in the incidence of primary central nervous system non-Hodgkin lymphoma: a Surveillance, Epidemiology, and End Results analysis. Cancer 2002;95:1504-1510.

30. Hochberg FH, Batchelor T, Loeffler JS. Clinical presentation, pathologic features, and diagnosis of primary central nervous system lymphoma. Available at: http://www.uptodate.com. Last updated: Nov. 13, 2008; last literature review version 17.1: January 2009. Accessed April 24, 2009.

31. Hormigo A, Abrey L, Heinemann MH, DeAngelis LM. Ocular presentation of primary central nervous system lymphoma: diagnosis and treatment. Br J Haematol 2004;126:202-208.

32. Park S, Abad S, Tulliez M, et al. Pseudouveitis: a clue to the diagnosis of primary central nervous system lymphoma in immunocompetent patients. Medicine (Baltimore) 2004;83:223-232.

33. Bühring U, Herrlinger U, Krings T, Thiex R, Weller M, Küker W. MRI features of primary central nervous system lymphomas at presentation. Neurology 2001;57:393-396.

34. Cha S, Knopp EA, Johnson G, et al. Intracranial mass lesions: dynamic contrast-enhanced susceptibility-weighted echo-planar perfusion MR imaging. Radiology 2002;223:11-29.

35. Guo AC, Cummings TJ, Dash RC, Provenzale JM. Lymphomas and high-grade astrocytomas: comparison of water diffusibility and histologic characteristics. Radiology 2002;224:177-183.

36. Huang MQ, Pickup S, Nelson DS, et al. Monitoring response to chemotherapy of non-Hodgkin's lymphoma xenografts by $\mathrm{T}_{2-}$ weighted and diffusion-weighted MRI. NMR Biomed 2008;21: 1021-1029.

37. Balmaceda C, Gaynor JJ, Sun M, Gluck JT, DeAngelis LM. Leptomeningeal tumor in primary central nervous system lymphoma: recognition, significance, and implications. Ann Neurol 1995;38: 202-209.

38. Batchelor T, Loeffler JS. Primary CNS lymphoma. J Clin Oncol 2006;24:1281-1288.

39. Fitzsimmons A, Upchurch K, Batchelor T. Clinical features and diagnosis of primary central nervous system lymphoma. Hematol Oncol Clin North Am 2005;19:689-703, vii.

40. Pierce MA, Johnson MD, Maciunas RJ, et al. Evaluating contrastenhancing brain lesions in patients with AIDS by using positron emission tomography. Ann Intern Med 1995;123:594-598.
41. Kulkarni AV, Guha A, Lozano A, Bernstein M. Incidence of silent hemorrhage and delayed deterioration after stereotactic brain biopsy. J Neurosurg 1998;89:31-35.

42. Boviatsis EJ, Kouyialis AT, Stranjalis G, Korfias S, Sakas DE. CT-guided stereotactic biopsies of brain stem lesions: personal experience and literature review. Neurol Sci 2003;24:97-102.

43. Soo TM, Bernstein M, Provias J, et al. Failed stereotactic biopsy in a series of 518 cases. Stereotact Funct Neurosurg 1995;64:183196.

44. Weller M. Glucocorticoid treatment of primary CNS lymphoma. J Neurooncol 1999;43:237-239.

45. National Comprehensive Cancer Network. NCCN clinical practice guidelines in oncology: central nervous system cancers. Available at: http://www.nccn.org/professionals/physician_gls/f_guidelines. asp. Accessed April 24, 2009.

46. Jahnke K, Hummel M, Korfel A, et al. Detection of subclinical systemic disease in primary CNS lymphoma by polymerase chain reaction of the rearranged immunoglobulin heavy-chain genes. J Clin Oncol 2006;24:4754-4757.

47. Ferreri AJ, Blay JY, Reni M, et al. Prognostic scoring system for primary CNS lymphomas: the International Extranodal Lymphoma Study Group experience. J Clin Oncol 2003;21:266-272.

48. Abrey LE, DeAngelis LM, Yahalom J. Long-term survival in primary CNS lymphoma. J Clin Oncol 1998;16:859-863.

49. Bellinzona M, Roser F, Ostertag H, Gaab RM, Saini M. Surgical removal of primary central nervous system lymphomas (PCNSL) presenting as space occupying lesions: a series of 33 cases. Eur J Surg Oncol 2005;31:100-105.

50. Henry JM, Heffner RR Jr, Dillard SH, Earle KM, Davis RL. Primary malignant lymphomas of the central nervous system. Cancer 1974;34:1293-1302.

51. Murray K, Kun L, Cox J. Primary malignant lymphoma of the central nervous system: results of treatment of 11 cases and review of the literature. J Neurosurg 1986;65:600-607.

52. DeAngelis LM. Current management of primary central nervous system lymphoma. Oncology (Williston Park) 1995;9:63-71; discussion 71, 75-76, 78.

53. Nelson DF, Martz KL, Bonner H, et al. Non-Hodgkin's lymphoma of the brain: can high dose, large volume radiation therapy improve survival? Report on a prospective trial by the Radiation Therapy Oncology Group (RTOG): RTOG 8315. Int J Radiat Oncol Biol Phys 1992;23:9-17.

54. Harder H, Holtel H, Bromberg JE, et al. Cognitive status and quality of life after treatment for primary CNS lymphoma. Neurology 2004;62:544-547.

55. Bessell EM, López-Guillermo A, Villá S, et al. Importance of radiotherapy in the outcome of patients with primary CNS lymphoma: an analysis of the CHOD/BVAM regimen followed by two different radiotherapy treatments. J Clin Oncol 2002;20:231-236.

56. DeAngelis LM, Yahalom J, Thaler HT, Kher U. Combined modality therapy for primary CNS lymphoma. J Clin Oncol 1992;10: 635-643.

57. Omuro AM, Ben-Porat LS, Panageas KS, et al. Delayed neurotoxicity in primary central nervous system lymphoma. Arch Neurol 2005;62:1595-1600.

58. DeAngelis LM, Seiferheld W, Schold SC, Fisher B, Schultz CJ; Radiation Therapy Oncology Group Study 93-10. Combination chemotherapy and radiotherapy for primary central nervous system lymphoma: Radiation Therapy Oncology Group Study 93-10. J Clin Oncol 2002;20:4643-4648.

59. Shah GD, Yahalom J, Correa DD, et al. Combined immunochemotherapy with reduced whole-brain radiotherapy for newly diagnosed primary CNS lymphoma. J Clin Oncol 2007;25:4730-4735.

60. Correa DD, Rocco-Donovan M, DeAngelis LM, et al. Prospective cognitive follow-up in primary CNS lymphoma patients treated with chemotherapy and reduced-dose radiotherapy. J Neurooncol 2009;91:315-321.

61. Poortmans PM, Kluin-Nelemans HC, Haaxma-Reiche H, et al High-dose methotrexate-based chemotherapy followed by consolidating radiotherapy in non-AIDS-related primary central nervous system lymphoma: European Organization for Research and Treatment of Cancer Lymphoma Group Phase II Trial 20962. J Clin Oncol 2003;21:4483-4488. 
62. Ervin T, Canellos GP. Successful treatment of recurrent primary central nervous system lymphoma with high-dose methotrexate. Cancer 1980;45:1556-1557.

63. Herbst KD, Corder MP, Justice GR. Successful therapy with methotrexate of a multicentric mixed lymphoma of the central nervous system. Cancer 1976;38:1476-1478.

64. O'Neill BP, O'Fallon JR, Earle JD, et al. Primary central nervous system non-Hodgkin's lymphoma: survival advantages with combined initial therapy? Int J Radiat Oncol Biol Phys 1995;33:663673.

65. Schultz C, Scott C, Sherman W, et al. Preirradiation chemotherapy with cyclophosphamide, doxorubicin, vincristine, and dexamethasone for primary CNS lymphomas: initial report of radiation therapy oncology group protocol 88-06. J Clin Oncol 1996;14:556564 .

66. Shapiro WR, Young DF, Mehta BM. Methotrexate: distribution in cerebrospinal fluid after intravenous, ventricular and lumbar injections. N Engl J Med 1975;293:161-166.

67. Glantz MJ, Cole BF, Recht L, et al. High-dose intravenous methotrexate for patients with nonleukemic leptomeningeal cancer: is intrathecal chemotherapy necessary? J Clin Oncol 1998;16:15611567.

68. Khan RB, Shi W, Thaler HT, DeAngelis LM, Abrey LE. Is intrathecal methotrexate necessary in the treatment of primary CNS lymphoma? J Neurooncol 2002;58:175-178.

69. Doolittle ND, Miner ME, Hall WA, et al. Safety and efficacy of a multicenter study using intraarterial chemotherapy in conjunction with osmotic opening of the blood-brain barrier for the treatment of patients with malignant brain tumors. Cancer 2000;88:637-647.

70. McAllister LD, Doolittle ND, Guastadisegni PE, et al. Cognitive outcomes and long-term follow-up results after enhanced chemotherapy delivery for primary central nervous system lymphoma. Neurosurgery 2000;46:51-60; discussion 60-51.

71. Cher L, Glass J, Harsh GR, Hochberg FH. Therapy of primary CNS lymphoma with methotrexate-based chemotherapy and deferred radiotherapy: preliminary results. Neurology 1996;46:17571759.

72. Guha-Thakurta N, Damek D, Pollack C, Hochberg FH. Intravenous methotrexate as initial treatment for primary central nervous system lymphoma: response to therapy and quality of life of patients. J Neurooncol 1999;43:259-268.

73. Batchelor T, Carson K, O'Neill A, et al. Treatment of primary CNS lymphoma with methotrexate and deferred radiotherapy: a report of NABTT 96-07. J Clin Oncol 2003;21:1044-1049.

74. Herrlinger U, Küker W, Uhl M, et al. NOA-03 trial of high-dose methotrexate in primary central nervous system lymphoma: final report. Ann Neurol 2005;57:843-847.

75. Reni M, Mason W, Zaja F, et al. Salvage chemotherapy with temozolomide in primary CNS lymphomas: preliminary results of a phase II trial. Eur J Cancer 2004;40:1682-1688.

76. Reni M, Zaja F, Mason W, et al. Temozolomide as salvage treatment in primary brain lymphomas. Br J Cancer 2007;96:864-867.

77. Coiffier B, Lepage E, Briere J, et al. CHOP chemotherapy plus rituximab compared with $\mathrm{CHOP}$ alone in elderly patients with diffuse large-B-cell lymphoma. N Engl J Med 2002;346:235-242.

78. Rubenstein JL, Combs D, Rosenberg J, et al. Rituximab therapy for CNS lymphomas: targeting the leptomeningeal compartment. Blood 2003;101:466-468.

79. Stemmler HJ, Schmitt M, Willems A, et al. Ratio of trastuzumab levels in serum and cerebrospinal fluid is altered in HER2-positive breast cancer patients with brain metastases and impairment of blood-brain barrier. Anticancer Drugs 2007;18:23-28.

80. Issa S, Shen A, Karch J, et al. Treatment of primary CNS lymphoma with induction high-dose methotrexate, temozolomide, rituximab followed by consolidation cytarabine/etoposide: a pilot study with biomarker analysis. Presented at the American Society of Hematology 2008 Annual Meeting; December 8, 2008; Atlanta, GA. Abstract 1364 .
81. Pels H, Schmidt-Wolf IG, Glasmacher A, et al. Primary central nervous system lymphoma: results of a pilot and phase II study of systemic and intraventricular chemotherapy with deferred radiotherapy. J Clin Oncol 2003;21:4489-4495.

82. Pels H, Juergens A, Glasmacher A, et al. Early relapses in primary CNS lymphoma after response to polychemotherapy without intraventricular treatment: results of a phase II study. J Neurooncol 2009;91:299-305.

83. Abrey LE, Moskowitz CH, Mason WP, et al. Intensive methotrexate and cytarabine followed by high-dose chemotherapy with autologous stem-cell rescue in patients with newly diagnosed primary CNS lymphoma: an intent-to-treat analysis. J Clin Oncol 2003;21: 4151-4156.

84. Montemurro M, Kiefer T, Schüler F, et al. Primary central nervous system lymphoma treated with high-dose methotrexate, high-dose busulfan/thiotepa, autologous stem-cell transplantation and response-adapted whole-brain radiotherapy: results of the multicenter Ostdeutsche Studiengruppe Hämato-Onkologie OSHO-53 phase II study. Ann Oncol 2007;18:665-671.

85. Soussain C, Hoang-Xuan K, Taillandier L, et al; Société Française de Greffe de Moëlle Osseuse-Thérapie Cellulaire. Intensive chemotherapy followed by hematopoietic stem-cell rescue for refractory and recurrent primary CNS and intraocular lymphoma: Société Française de Greffe de Moëlle Osseuse-Thérapie Cellulaire. J Clin Oncol 2008;26:2512-2518.

86. Plotkin SR, Betensky RA, Hochberg FH, et al. Treatment of relapsed central nervous system lymphoma with high-dose methotrexate. Clin Cancer Res 2004;10:5643-5646.

87. Herrlinger U, Brugger W, Bamberg M, Küker W, Dichgans J, Weller M. PCV salvage chemotherapy for recurrent primary CNS lymphoma. Neurology 2000;54:1707-1708.

88. Nguyen PL, Chakravarti A, Finkelstein DM, et al. Results of whole-brain radiation as salvage of methotrexate failure for immunocompetent patients with primary CNS lymphoma. J Clin Oncol 2005;23:1507-1513.

89. Sakamoto M, Oya N, Mizowaki T, et al. Initial experiences of palliative stereotactic radiosurgery for recurrent brain lymphomas. J Neurooncol 2006;77:53-58.

90. Rubenstein JL, Fridlyand J, Abrey L, et al. Phase I study of intraventricular administration of rituximab in patients with recurrent CNS and intraocular lymphoma. J Clin Oncol 2007;25:13501356.

91. Chamberlain MC, Johnston SK, Van Horn A, Glantz MJ. Recurrent lymphomatous meningitis treated with intra-CSF rituximab and liposomal ara-C. J Neurooncol 2009;91:271-277.

92. de Smet MD, Stark-Vancs V, Kohler DR, Smith J, Wittes R, Nussenblatt RB. Intraocular levels of methotrexate after intravenous administration. Am J Ophthalmol 1996;121:442-444.

93. Batchelor TT, Kolak G, Ciordia R, Foster CS, Henson JW. Highdose methotrexate for intraocular lymphoma. Clin Cancer Res 2003;9:711-715.

94. Baumann MA, Ritch PS, Hande KR, et al. Treatment of intraocular lymphoma with high-dose Ara-C. Cancer 1986;57:1273-1275.

95. Grimm SA, McCannel CA, Omuro AM, et al. Primary CNS lymphoma with intraocular involvement: International PCNSL Collaborative Group Report. Neurology 2008;71:1355-1360.

96. Itty S, Pulido JS. Rituximab for intraocular lymphoma. Retina 2009;29:129-132.

97. Kim H, Csaky KG, Chan CC, et al. The pharmacokinetics of rituximab following an intravitreal injection. Exp Eye Res 2006; $82: 760-766$

98. Ohguro N, Hashida N, Tano Y. Effect of intravitreous rituximab injections in patients with recurrent ocular lesions associated with central nervous system lymphoma. Arch Ophthalmol 2008;126: 1002-1003.

99. Rubenstein JL, Shen A, Batchelor TT, Kadoch C, Treseler P, Shuman MA. Differential gene expression in central nervous system lymphoma. Blood 2009;113:266-267. 